\title{
Análise da efetividade da terapia nutricional em pacientes sob cuidados paliativos do programa Melhor em Casa no município de Queimadas-PB
}

Analysis of the effectiveness of nutritional therapy in patients under palliative care of the Best at Home program in the municopality of Queimadas - PB

Análisis de la efectividad de la terapia nutricional em pacientes bajo cuidados paliativos del programa Mejor em Casa em el município de Queimadas - PB

\section{Resumo}

O Programa Melhor em Casa visa acompanhar com serviços especializados o paciente no aconchego do seu lar que é um ambiente mais saudável. A Terapia Nutricional é um conjunto de métodos de cuidados para manutenção ou recuperação do estado nutricional do paciente através da nutrição parenteral ou enteral, esta deve ser bem acompanhada para o restabelecimento da saúde da pessoa assistida. Assim, a presente pesquisa procurou avaliar a efetividade da terapia nutricional enteral nos pacientes em cuidados paliativos assistidos por este programa, no município de Queimadas-PB. Foi aplicado um estudo do tipo descritivo, qualitativo e quantitativo, onde a população foi composta por pacientes adultos e idosos, com patologias diversas. As informações foram coletadas dos prontuários com posterior análise dos registros em fichas individuais. Foram avaliados 10 pacientes, com idade média de 68,30 anos. O baixo peso e as afecções neurológicas estiveram presentes em $50 \%$ dos pacientes. Em relação à via de acesso $50 \%$ possuíam prescrição de sonda nasogástrica. Quanto à média do percentual de adequação proteica e de adequação energética ambas foram alcançadas. Embora a média geral de ingestão calórica e protéica tenham atendido as recomendações preconizadas, foi verificado uma incidência de baixo peso em metade dos indivíduos, o que pode ser justificada pelo surgimento de sintomas gastrointestinais, inadequação na administração da dieta ou avanço das patologias.

Palavras-chave: Dietoterapia; Assistência domiciliar; Cuidados paliativos. 


\begin{abstract}
The Best at Home Program aims to accompany the patient with specialized services in the comfort of their home, which is a healthier environment. Nutritional Therapy is a set of care methods for maintaining or recovering the patient's nutritional status through Parenteral or Enteral Nutrition, this must be well monitored to restore the health of the assisted person. Thus, the present research sought to evaluate the effectiveness of enteral nutritional therapy in patients in palliative care assisted by this program, in the municipality of Queimadas-PB. A descriptive, qualitative and quantitative study was applied, where the population was composed of adult and elderly patients, with different pathologies. The information was collected from the medical records with subsequent analysis of the records in individual files. Ten patients were evaluated, with an average age of 68.30 years. Low weight and neurological disorders were present in 50\% of patients. Regarding the access route, 50\% had a nasogastric tube prescription. As for the average percentage of protein adequacy and energy adequacy, both were achieved. Although the overall average caloric and protein intake met the recommended recommendations, an incidence of low weight was found in half of the individuals, which can be justified by the appearance of gastrointestinal symptoms, inadequate dietary management or progression of pathologies.
\end{abstract}

Keywords: Diet therapy; Home nursing; Palliative care.

\title{
Resumen
}

El Programa Best at Home tiene como objetivo acompañar al paciente con servicios especializados en la comodidad de su hogar, que es un entorno más saludable. La Terapia Nutricional es un conjunto de métodos de cuidado para mantener o recuperar el estado nutricional del paciente a través de la nutrición parenteral o enteral, esta debe estar bien monitoreada para restaurar la salud de la persona asistida. Así, la presente investigación buscó evaluar la efectividad de la terapia nutricional enteral en pacientes en cuidados paliativos asistidos por este programa, en el municipio de Queimadas-PB. Se aplicó un estudio descriptivo, cualitativo y cuantitativo, donde la población estuvo compuesta por pacientes adultos y ancianos, con diferentes patologías. La información se recopiló de los registros médicos con el análisis posterior de los registros en archivos individuales. Se evaluaron diez pacientes, con una edad media de 68,30 años. El bajo peso y los trastornos neurológicos estuvieron presentes en el 50\% de los pacientes. En cuanto a la vía de acceso, el 50\% tenía prescripción de sonda nasogástrica. En cuanto al porcentaje medio de adecuación proteica y adecuación energética, se lograron ambos. Si bien la ingesta calórica y proteica promedio global cumplió con las recomendaciones recomendadas, se encontró una incidencia de bajo peso en la mitad de los individuos, lo que puede justificarse por la aparición de síntomas gastrointestinales, manejo dietético inadecuado o progresión de patologías.

Palabras clave: Dietoterapia; Atención domiciliaria de salud; Cuidados paliativos.

\section{Introdução}

As mudanças clínicas referentes à deglutição e/ou integridade do trato digestório de indivíduos são percebidas pelo aumento da expectativa de vida, crescimento de doenças crônicas, elevado número de vítimas de acidentes no trânsito e aos casos de violência. Assim, em alguns casos específicos é preciso que a alimentação ocorra por meio de procedimentos que substituam ou complementem a nutrição por via oral. A maioria dos pacientes hospitalizados se encontra em situação aguda que em muitos casos interfere no estado nutricional. A desospitalização é feita conforme o quadro clínico, ou seja, quando o enfermo estiver com condições de receber assistência domiciliar (Brasil, 2015; Lima et al., 2018).

A terapia nutricional $(\mathrm{TN})$ é um conjunto de métodos terapêuticos para manutenção ou recuperação do estado nutricional do enfermo através da Nutrição Parenteral ou Enteral. Dessa forma, é capaz de prorrogar a sobrevida, diminuir a redução de peso e tornar melhor a qualidade de vida de pessoas neste tipo de cuidado, através da redução dos efeitos adversos, dos tratamentos e/ou das enfermidades (Brasil, 2000; Morais et al., 2016). Nesse caso, a implementação da TN, que é reconhecida e utilizada como parte da atenção hospitalar, precisa ser oferecida no âmbito domiciliar de forma qualificada e organizada (Brasil, 2015).

A nutrição enteral (NE) é uma forma de ofertar nutrientes ao trato digestório por via de uma sonda. As fórmulas enterais são compostas por carboidratos, lipídios, proteínas, água, fibras, vitaminas e minerais. Na NE as vias de acesso podem ser realizadas no estômago, duodeno ou jejuno. Nos pacientes que requerem este tipo de terapia por um curto tempo (menos de seis semanas), a sonda nasoenteral é a mais aplicada, em virtude da facilidade do posicionamento e seu baixo valor. Quando a TN ultrapassa quatro semanas, a gastrostomia e a jejunostomia são recomendadas (Cuppari, 2019). 
O Programa Melhor em Casa (PMC) criado pelo Governo Federal oferece serviços de atenção domiciliar (AD), com equipes de diversos profissionais da saúde, para proporcionar a recuperação do paciente no aconchego do seu lar que é um ambiente mais saudável. Além disso, possibilita a desocupação de leitos hospitalares (Nishimura, Carrara, \& Freitas, 2019). A equipe multiprofissional deve capacitar e supervisionar os cuidadores dos indivíduos em atendimento domiciliar. Dessa forma, o vínculo entre os profissionais, familiares, cuidadores e pacientes são essenciais para efetivação da AD (Brasil, 2012).

O indivíduo em atendimento domiciliar pode estar em estado de desnutrição ou ficar desnutrido no decorrer da assistência (Leydon \& Dahl, 2008). Tal situação é agravada pela falta de atenção dos doentes, familiares e profissionais da área de saúde. A desnutrição eleva o risco de infecção, internação hospitalar e mortes, além disso, modifica a qualidade de vida (Arvanitakis et al., 2008).

$\mathrm{Na}$ maior parte das situações, os enfermos são analisados para o recebimento da terapia nutricional domiciliar no âmbito hospitalar como também podem ser identificados nos consultórios médicos. A equipe de nutrição ou médica do hospital é quem estabelece a Terapia Nutricional Enteral Domiciliar (TNED) antes da sua volta para o domicílio (Aanholt et al., 2012). A intervenção nutricional em cuidados paliativos tem que considerar as determinações do paciente e de seus parentes e os princípios bioéticos como: autonomia, não maleficência, beneficência e justiça (Morais et al., 2016).

O Nutricionista exerce um papel essencial nos cuidados paliativos, visto que, os pacientes vivenciam sintomas que acometem a via de alimentação, assim consideram-se as necessidades nutricionais, preferências e hábitos alimentares do indivíduo, para controlar os sintomas bem como garantir satisfação e conforto (Benarroz, Faillace, \& Barbosa, 2009). É primordial, respeitar a vontade do indivíduo, seja qual for à conduta nutricional a ser realizada. Dessa maneira, favorecendo a qualidade de vida do paciente. Além disso, este profissional fornece esclarecimentos aos pacientes e familiares sobre o tratamento (Corrêa \& Shibuya, 2007).

Nesta perspectiva a Atenção Nutricional incluir-se no cuidado integral nas redes de atenção à saúde (RAS), compreendendo os cuidados na alimentação e nutrição que devem estar relacionados às outras ações de atenção à saúde do Sistema Único de Saúde (SUS), para pacientes, famílias, comunidades, colaborando para a formação de uma rede integrada, decisória e humanizada de cuidados (BRASIL, 2013).

Devido ao crescente número de usuários da assistência domiciliar que necessitam de cuidados nutricionais específicos, em virtude de determinadas situações que comprometem a alimentação e interferem no estado nutricional, a TN é essencial para promover a melhora na qualidade de vida através de um atendimento humanizado. Portanto, o objetivo desse estudo foi analisar a efetividade da terapia nutricional enteral nos pacientes em cuidados paliativos assistidos pelo Programa Melhor em Casa promovido no município de Queimadas - PB.

\section{Metodologia}

O estudo realizado foi do tipo descritivo, qualitativo e quantitativo, com emprego de dados dos pacientes sob cuidados paliativos do programa Melhor em Casa, no qual é composto por uma Equipe Multidisciplinar de Atenção Domiciliar - EMAD (médico, enfermeiro, fisioterapeuta, técnico de enfermagem) e pela Equipe Multidisciplinar de apoio - EMAP (nutricionista, psicólogo, assistente social, odontólogo). A pesquisa foi realizada no município de Queimadas-PB, junto a Secretaria de Saúde do Município.

A coleta de dados foi realizada por meio de análise de prontuários no período de setembro a dezembro de 2020 . A amostra foi constituida por pacientes adultos e idosos, com patologias diversas, de ambos os sexos, em TNE exclusiva, cadastrados no PMC. Os pacientes com alimentação por via oral, menores de 18 anos e que não estivessem nos critérios de inclusão descritos anteriormente foram excluídos. O programa atende um total de 67 pacientes, destes, foram selecionados 10 pacientes baseados nas informações contidas nos prontuários, tais como via de alimentação e idade. 
A mesma foi realizada a partir das informações contidas nos prontuários dos pacientes, no período de setembro a dezembro de 2020, utilizando como instrumento uma ficha de coleta de dados produzida pelos pesquisadores do presente estudo, para obtenção das informações. As variáveis analisadas foram: sexo; idade; patologia; via de acesso da TNE; tipo de fórmula; data de cadastro do paciente; data do início da TNE; diagnóstico nutricional; complicações gastrointestinais; recomendação, prescrição, infusão de energia e de proteína.

As informações em relação ao consumo alimentar dos pacientes foram obtidas através do recordatório alimentar de 24 horas, coletado pelo nutricionista responsável do programa, e posteriormente realizado o somatório total de calorias e proteínas pela pesquisadora utilizando o programa Dietbox. Para as análises dos dados as informações foram compiladas em uma planilha do Microsoft Excel 365@, para apoio, registro dos dados e cálculo dos percentuais. O mesmo programa foi utilizado para montagem das figuras apresentadas nos resultados.

$\mathrm{Na}$ análise dos dados estatísticos referente as recomendações, infusões e adequações calóricas e proteicas foi utilizado o programa - Statistics Analys Systems, para determinação das médias e desvio padrão (SAS, 1999). A adequação da oferta calórico-proteica foi calculada através da relação percentual entre o valor ingerido e o recomendado. Foi utilizado como referencial a ser atingido o valor de 80\% de adequação (Ribeiro, 2015).

Para tanto, a pesquisa foi autorizada pelo local com assinatura de uma Autorização da Instituição Concedente ou Carta de Anuência pelo Secretário de Saúde do Município, como também foi instituído um Termo de Autorização para Pesquisa em Arquivo e/ou Prontuários, já que foi o meio utilizado no estudo. O projeto está de acordo com a Resolução nº 466/2012 do Conselho Nacional de Saúde no Ministério da Saúde que dispõe diretrizes que regulamentam as pesquisas envolvendo seres humanos de forma direta ou indireta. A coleta de dados foi iniciada mediante autorização do Comitê de Ética em pesquisa do Centro Universitário da UniFacisa, assegurando sigilo das informações coletadas e o direito dos indivíduos retirarem o consentimento a qualquer momento da pesquisa. A mesma foi submetida em julho de 2020 e recebeu o parecer de número 4.180.557 no mesmo período.

\section{Resultados e Discussão}

Os resultados obtidos na pesquisa para a caracterização de sexo, fórmulas, tipo de acesso e patologias são demonstrados na Tabela 1 com apresentação das variáveis, percentual encontrado e o número amostral correspondente.

Dos 10 pacientes avaliados, $30 \%$ foram do sexo masculino e $70 \%$ feminino. A distribuição do sexo apresentou predominância do gênero feminino o que se assemelha com o estudo de Azank et al. (2009) realizado no estado de São Paulo com pacientes em uso de TNE, atendidos pelo Serviço de Assistência Domiciliar (SAD) no qual a maioria foram mulheres $(66,6 \%)$.

A idade média foi de 68,30 anos $( \pm 24,25)$, variando entre 18 e 97 anos, sendo evidenciada uma prevalência de idosos. Em um estudo semelhante realizado em Foz do Iguaçu (PR) com usuários de nutrição enteral domiciliar, a idade média foi de 73 anos (Fuhr \& Ciachi, 2019). Esse resultado difere com um trabalho envolvendo pacientes em nutrição enteral domiciliar que apontam uma média de idade de 77 anos (Lim et al., 2019).

O principal tipo de dieta oferecida foram as fórmulas nutricionais mistas hipercalórica e hiperproteica, representando $80 \%$ dos pacientes atendidos pelo programa. Este resultado difere do encontrado em um estudo conduzido com pacientes em uso de TNED, atendidos pelo Programa de Terapia Nutricional Enteral Domiciliar (PTNED), no qual foi verificado que 98,3\% dos pacientes utilizavam a fórmula normocalórica e normoproteica (Menezes \& Fortes, 2019). Vale ressaltar que o tipo de dieta utilizada depende de vários fatores como diagnóstico clínico, estado nutricional e a via de administração da dieta. 
Tabela 1. Caracterização dos pacientes em uso de TNE excluiva, Queimadas-PB, Brasil, 2020.

\begin{tabular}{|c|c|}
\hline Variáveis & $\mathrm{N}=\mathbf{1 0}$ \\
\hline Sexo - & n (\%) \\
\hline Masculino & $3(30 \%)$ \\
\hline Feminino & $7(70 \%)$ \\
\hline Idade - média \pm DP & $68,30 \pm 24,25$ \\
\hline Fórmulas - & n (\%) \\
\hline Hipercalórica e hiperproteica & $8(80 \%)$ \\
\hline Normocalórica e hiperproteica & $2(20 \%)$ \\
\hline Tipo de acesso - & n (\%) \\
\hline Nasogástrica & $5(50 \%)$ \\
\hline Nasoentérica & $4(40 \%)$ \\
\hline Gastrostomia & $1(10 \%)$ \\
\hline Patologias - (paciente pode conter mais de uma patologia) & n (\%) \\
\hline Afecções neurológicas & $5(50 \%)$ \\
\hline Problemas respiratórios & $4(40 \%)$ \\
\hline Câncer & $3(30 \%)$ \\
\hline Hipertensão & $3(30 \%)$ \\
\hline Diabetes mellitus tipo 2 & $2(20 \%)$ \\
\hline Osteoporose & $1(10 \%)$ \\
\hline
\end{tabular}

Fonte: Autores (2021).

Referente à via de acesso, 50\% tinha prescrição de sonda nasogástrica (SNG), $40 \%$ sonda nasoenteral (SNE) e $10 \%$ gastrostomia (GTT). Os resultados encontrados por Menezes e Fortes (2019), evidenciou que a maioria dos casos utilizavam a SNE/SNG (62,4\%), seguida pela GTT (36,2\%). Outro estudo, feito por Azank et al. (2009), constatou que (73,3\%) receberam a SNE e $(26,6 \%)$ GTT.

Observou-se que os principais diagnósticos clínicos foram doenças neurológicas, seguido de problemas respiratórios, câncer, hipertensão, diabetes mellitus tipo 2 e osteoporose. Dentre os distúrbios neurológicos encontrados neste estudo pode-se citar a demência senil, epilepsia, doença de Alzheimer e sequelas neurológicas pós-parada cardiorrespiratória prolongada. Que demandam um cuidado maior e em alguns casos por toda a vida do paciente. Verificou-se nos prontuários analisados que a maioria dos pacientes não apresentava apenas um diagnóstico, mas várias doenças, manifestando, assim, mais de uma patologia.

Em estudo realizado por Menezes e Fortes (2019), as doenças neurológicas corresponderam a 57,2\%, seguido de câncer (22,5\%). Outro estudo semelhante de Brondani et al. (2013) com pacientes de um serviço de internação domiciliar em Santa Maria (RS), as patologias predominante foram as desordens neurológicas (31,5\%) e câncer (18\%). Esse resultado difere do estudo de Martelli et al. (2011), realizado com pacientes do Programa de Internação Domiciliar HU em Casa, em Montes Claros (MG), nos quais houve predomínio de doenças respiratórias (19\%).

Na Figura 1, encontram-se as complicações gastrointestinais dos pacientes avaliados no estudo. Foi possível verificar que de todos os pacientes pesquisados $30 \%$ nao apresentaram complicações gastrointestinais, (40\%) apresentaram diarreia com frequencia, (20\%) apresentaram constipação e (10\%) distensão abdominal. 
Figura 1. Apresentação das complicações gastrointestinais dos pacientes avaliados.

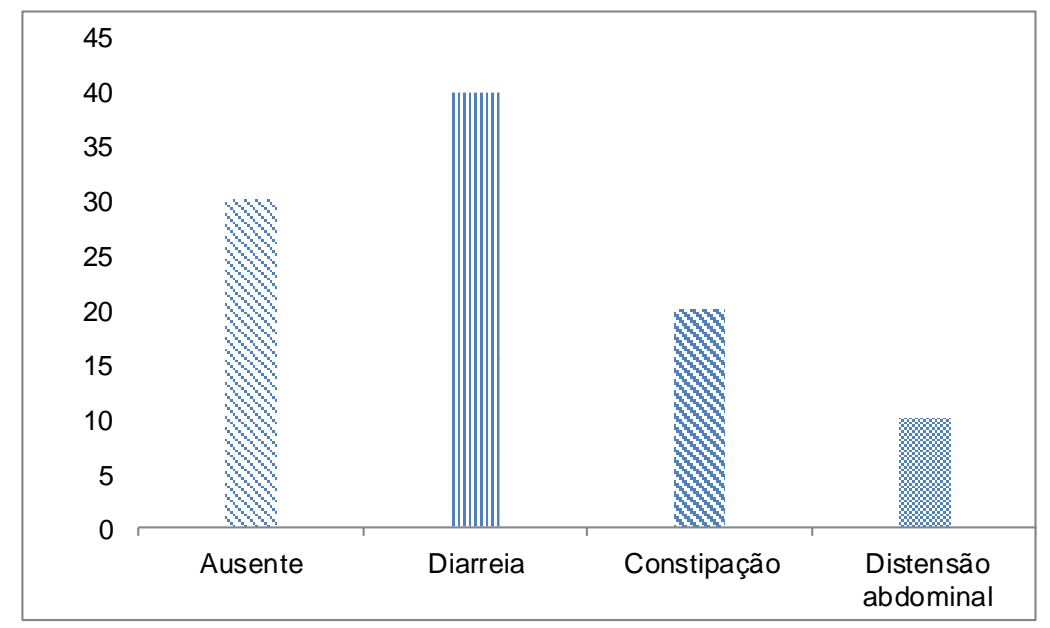

Fonte: Autores (2021).

Embora seja observada uma gravidade nutricional em alguns enfermos, é importante ressaltar que a finalidade da terapia paliativa é proporcionar conforto e qualidade de vida ao paciente. Assim sendo, estratégias nutricionais para diminuir os sintomas gastrointestinais tem o propósito de melhorar a qualidade de vida, bem como conceder condições para que o doente se alimente por via oral. É fundamental saber o prognóstico da patologia e a expectativa de vida do paciente, entendendo a fragilidade do processo, visando proporcionar a acolhida do paciente, parentes e cuidadores, através de uma linguagem clara que ressalte informações do enfermo (Carvalho et al., 2018).

A pesquisa de Lim et al. (2018) constataram que as complicações mais comumente presentes foram constipação (31\%), distensão abdominal (28\%) e vômitos (22\%). Já no estudo de Medeiros et al. (2019) realizado com pacientes internados em uma unidade de terapia intensiva de um hospital público da cidade de Rio Branco (AC), foi observada a ocorrência de sintomas gastrointestinais em $65,3 \%$ dos pacientes, sendo caracterizados por constipação (20,8\%), diarreia (18,1\%) e distensão abdominal (8,3\%). Vale ressaltar a importância de identificar a prevalência desses acontecimentos, para que os mesmos não influenciem na terapia nutricional.

Quanto ao estado nutricional, observou-se prevalência de baixo peso (50\%), seguido de eutrofia (40\%), sobrepeso (10\%). Esses dados podem ser melhor acompanhado na Figura 2.

Figura 2. Distribuição do estado nutricional dos pacientes avaliados.

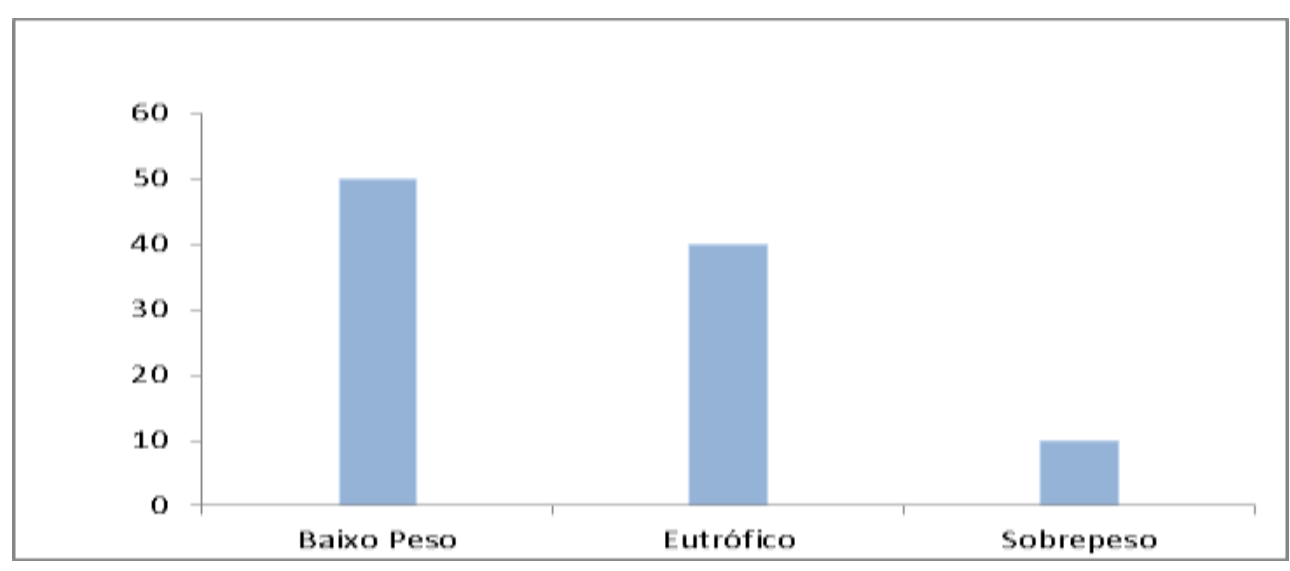

Fonte: Autores (2021). 
Esses dados são preocupantes visto que, a desnutrição manifesta inúmeras complicações para o paciente, estando relacionada ao aumento de reinternações hospitalares, bem como uma maior morbidade e mortalidade (Arvanitakis, 2008).

Os dados encontrados corroboram com os de Ortega et al. (2017) realizado em Jaguariúna (SP) com pacientes adultos e idosos, inclusos no Programa Melhor em Casa que utilizavam exclusivamente dieta enteral, na qual foi verificado que 50\% estavam com baixo peso, 33,33\% eutrofia, 16,66\% sobrepeso. Resultado semelhante foi encontrado no estudo de Gambato e Boscaini (2015), no qual 39,5\% dos indivíduos apresentaram baixo peso, 30,7\% eutrofia e 29,8\% excesso de peso.

Na Tabela 2, encontram-se detalhados os valores referentes às recomendações e infusões calóricas e proteicas, e suas respectivas adequações. A média das necessidades calóricas do presente estudo foi de $1878,80 \mathrm{Kcal} / \mathrm{dia}( \pm 276,07)$ e as necessidades proteicas de 84,80g/dia $( \pm 18,28)$ semelhante aos achados por Medeiros et al. (2019), realizado no hospital público da cidade de Rio Branco - Acre no qual os pesquisadores obtiveram a média de 1776,3 Kcal/dia $( \pm 256,9)$ e de recomendação proteica 89,7 g/dia $( \pm 12,78)$. Enquanto que os valores obtidos em estudo de Rocha e Jorge (2011) realizado no Hospital da Universidade Federal de Goiás - GO, foi de 1438,2 Kcal/dia ( $\pm 342,2)$.

Tabela 2. Caracterização das recomendações, infusões e adequações calóricas e proteicas, Queimadas-PB, Brasil, 2020.

\begin{tabular}{ccc}
\hline & Média & Desvio Padrão \\
\hline Recomendação calórica & $1878,80 \mathrm{kcal}$ & 276,07 \\
Recomendação Proteica & $84,80 \mathrm{~g}$ & 18,28 \\
Infusão energética & $1533,78 \mathrm{kcal}$ & 364,32 \\
Infusão proteica & $77,55 \mathrm{~g}$ & 21,28 \\
Adequação energética & $82,91 \%$ & 21,08 \\
Adequação de PTN & $91,53 \%$ & 15,86 \\
\hline
\end{tabular}

Fonte: Autores (2021).

Na terapia nutricional enteral, Ribeiro (2015) considera adequada uma ingestão calórica e proteica, quando é atingido $80 \%$ de infusão das necessidades nutricionais. No presente estudo a média do percentual de adequação proteica (91,53\%) foi maior em relação à adequação energética $(82,91 \%)$, assim, ambas as metas foram atingidas.

Com relação às adequações, foi verificado que $60 \%$ dos pacientes receberam aporte calórico adequado. Já a análise proteica revelou uma adequação para $80 \%$ dos pacientes. No estudo realizado por Medeiros et al. (2019) demonstraram que 79,2\% dos pacientes apresentaram adequação energética, enquanto na pesquisa de Lins et al. (2015) foi verificada uma adequação proteica inferior de $64,4 \%$ na população estudada.

Na terapia nutricional enteral, Ribeiro (2015) considera adequada uma ingestão calórica e proteica, quando é atingido $80 \%$ de infusão das necessidades nutricionais. No presente estudo a média do percentual de adequação proteica $(91,53 \%)$ foi maior em relação à adequação energética $(82,91 \%)$, assim, ambas as metas foram atingidas.

Com relação às adequações, foi verificado que $60 \%$ dos pacientes receberam aporte calórico adequado. Já a análise proteica revelou uma adequação para $80 \%$ dos pacientes. No estudo realizado por Medeiros et al. (2019) demonstraram que 79,2\% dos pacientes apresentaram adequação energética, enquanto na pesquisa de Lins et al. (2015) foi verificada uma adequação proteica inferior de $64,4 \%$ na população estudada.

Com isso, o aporte nutricional planejado em relação ao realizado mostrou-se eficiente na terapia acompanhada pelo programa assistencial. É importante ressaltar que existe em alguns casos específicos uma inadequação na elaboração da dieta, na qual é feita pelos cuidadores ou pacientes, podendo ser explicada pela ausência de conhecimento, como também pela falta 
de atenção, a partir disso é realizado um treinamento pela equipe do programa Melhor em Casa para melhor adesão a manipulação da dieta para que não ocorra nenhuma intercorrência.

Lembrando que o papel do nutricionista neste momento é de suma importância, haja visto que, por meio das prescrições e orientações nutricionais para os pacientes e cuidadores tem principalmente como objetivo adequar os nutrientes conforme as necessidades do paciente, além de prevenir ou reduzir risco a saúde do indivíduo; prevenir ou fornecer auxílio na cicatrização de úlceras por pressão, prevenir ou reverter casos de desnutrição, prevenir ou controlar as doenças crônico degenerativas (Brasil, 2018).

Vale salientar que o estudo foi realizado com dados de prontuários de pacientes em atendimento domiciliar, diferente de alguns estudos realizados frequentemente em UTI, no qual se tem um monitoramento de maneira constante da evolução do paciente.

\section{Conclusão}

Observou-se que embora a média geral de ingestão calórica e protéica tenha atendido ao preconizado, foi verificado uma incidência de baixo peso em metade dos indivíduos dessa pesquisa, o que pode ser justificada pelo surgimento de sintomas gastrointestinais, inadequação na administração da dieta ou avanço das patologias. Vale ressaltar a importância da monitorização rotineira do valor nutricional infundido bem como das possíveis complicações, a fim de garantir maior adequação da dieta e consequentemente melhora no percentual do estado nutricional. Portanto, os resultados sugerem possíveis soluções para minimizar as intercorrências como reuniões e treinamentos para os cuidadores e/ou pacientes de forma periódica, bem como supervisão da execução dos cuidados a fim de avaliar a compreensão das informações, padronizando assim o manejo da terapia nutricional enteral domiciliar.

\section{Referências}

Aanholt, D. P. J. V. et al. (2012). Terapia nutricional domiciliar. Revista da Associação Médica Brasileira 58 (4), $408-411$.

Arvanitakis, M. et al. (2008). Nutrition in care homes and home care: how to implement adequate strategies (report of the Brussels Forum (22-23). Clinical nutrition (Edinburgh, Scotland), 27(4), 481-488.

Azank, A. T. et al. (2009). Indicadores nutricionais em pacientes alimentados por sonda, em sistema de "Home Care". Arquivos Catarinenses de Medicina, $38(4), 11-18$.

Benarroz, M. O., Faillace, G. B. D., \& Barbosa, L. A. (2009). Bioética e nutrição em cuidados paliativos oncológicos em adultos. Cadernos de Saúde Pública, 25(9), 1875-1882.

Brasil (2000). Resolução $n^{\circ}$ 63, de 06 de julho de 2000. Aprova o Regulamento Técnico para a Terapia de Nutrição Enteral. Ministério da saúde.

Brasil (2013). Ministério da Saúde. Secretaria de Atenção à Saúde. Departamento de Atenção Básica. Caderno de atenção domiciliar / Ministério da Saúde, Secretaria de Atenção à Saúde, Departamento de Atenção Básica. Ministério da Saúde.

Brasil (2012). Ministério da Saúde. Secretaria de Atenção à Saúde. Departamento de Atenção Básica. Caderno de atenção domiciliar / Ministério da Saúde, Secretaria de Atenção à Saúde, Departamento de Atenção Básica. Ministério da Saúde.

Brasil (2015). Ministério da Saúde. Secretaria de Atenção à Saúde. Departamento de Atenção Básica. Cuidados em terapia nutricional / Ministério da Saúde, Secretaria de Atenção à Saúde, Departamento de Atenção Básica. Ministério da Saúde.

Brasil (2018). Protocolo de Atenção à Saúde. Nutrição na Atenção Domiciliar. Governo do Distrito Federal.

Brondani, C. M. et al. (2013). Caracterização de pacientes dependentes de tecnologias de um serviço de internação domiciliar. Revista de Enfermagem da UFSM, 3, $689-699$.

Carvalho, R. T. et al. (2018). Manual da residência de cuidados paliativos. Manole.

Corrêa, P. H., \& Shibuya, E. (2007). Administração da Terapia Nutricional em Cuidados Paliativos. Revista Brasileira de Cancerologia, 53 (3), $317-323$.

Cuppari, L. (2019). Nutrição clínica no adulto. (4a ed.), Manole.

Führ, A., \& Ciacchi, É. (2019). Possibilidades e Limitações da Terapia Nutricional Enteral na Compreensão de Cuidadores e Profissionais de uma Rede Pública de Saúde em Região de Fronteira. Demetra: Alimentação, Nutrição \& Saúde, 14, e36926. 
Research, Society and Development, v. 10, n. 6, e2410615232, 2021

(CC BY 4.0) | ISSN 2525-3409 | DOI: http://dx.doi.org/10.33448/rsd-v10i6.15232

Gambato, J, \& Boscaini, C. (2015). Adequação da prescrição dietética e sua associação com intercorrências em pacientes em uso de terapia nutricional enteral. Revista Brasileira de Nutrição Clínica, 30(4):330-43.

Leydon, N., \& Dahl, W. (2008). Improving the nutritional status of elderly residents of long-term care homes. Journal of health services research \& policy, 13 Suppl 1, 25-29.

Lim, M. L. et al. (2018). Caring for patients on home enteral nutrition: Reported complications by home carers and perspectives of community nurses. Journal of clinical nursing, 27(13-14), 2825-2835.

Lima, V. C. O. et al. (2018). Nutrição clínica. Sagah.

Lins, N.F. et al. (2015). Adequação da terapia nutricional enteral em pacientes críticos de um centro de referência em Pernambuco. Revista Brasileira de Nutrição Clínica, 1(30), 76-81.

Martelli, D. R. B. et al. (2011). Internação domiciliar: o perfil dos pacientes assistidos pelo Programa HU em Casa. Physis: Revista de Saúde Coletiva, 21(1), $147-157$.

Medeiros, I. M. S. et al. (2019). Caracterização e adequação energético-proteica da nutrição enteral em pacientes em uma unidade de terapia intensiva: Nutrição enteral no paciente crítico. South American Journal of Basic Education, Technical and Technological, 6(2), 381-395.

Menezes, C. S., \& Fortes, R. C. (2019). Estado nutricional e evolução clínica de idosos em terapia nutricional enteral domiciliar: uma coorte retrospectiva. Revista Latino-Americana de Enfermagem, 27, e3198.

Morais, S. R. et al. (2016). Nutrição, qualidade de vida e cuidados paliativos: uma revisão integrativa. Revista Dor, 17(2), 136-140.

Nishimura, F., Carrara, A. F., \& Freitas, C. E. (2019). Efeito do programa Melhor em Casa sobre os gastos hospitalares. Revista de Saúde Pública, 53, 104.

Ortega, N. C. et al. (2017). Análise da avaliação nutricional de participantes assistidos no programa "Melhor em Casa" com o uso de dieta enteral industrializada. Revista Intellectus, 1 (42).

Ribeiro, P. C. (2015). Nutrição. Atheneu.

Rocha, M. B. S., \& Jorge, A. M. V. (2011). Caracterização, adequação energética, proteica e progressão da dieta enteral em adultos hospitalizados. Revista Brasileira de Nutrição Clínica, 1 (30), 30-35.

SAS. (1999). Statistical analysis system. Usei Guid. Cary: SAS Institute. 